\title{
Sextant
}

Revue de recherche interdisciplinaire sur le genre et la sexualité

$37 \mid 2020$

Le rideau déchiré

\section{Les Kinsey des deux Allemagnes : recherche empirique en sexologie, enquêtes sur la sexualité et normalisation de la sexualité de la jeunesse pendant la guerre froide}

\section{Lutz Sauerteig}

\section{(2) OpenEdition}

1 Journals

Édition électronique

URL : https://journals.openedition.org/sextant/329

DOI : $10.4000 /$ sextant.329

ISSN : 2795-8736

Éditeur

Éditions de l'Université de Bruxelles

\section{Édition imprimée}

Date de publication : 1 décembre 2020

Pagination : $55-78$

ISBN : 978-2-8004-1744-8

ISSN : $1370-267 X$

Référence électronique

Lutz Sauerteig, "Les Kinsey des deux Allemagnes : recherche empirique en sexologie, enquêtes sur la sexualité et normalisation de la sexualité de la jeunesse pendant la guerre froide », Sextant [En ligne], 37 | 2020, mis en ligne le 01 novembre 2021, consulté le 24 janvier 2022. URL : http:// journals.openedition.org/sextant/329; DOI : https://doi.org/10.4000/sextant.329

\section{c) (†) (2)}

La revue Sextant est mise à disposition selon les termes de la Licence Creative Commons Attribution Pas d'Utilisation Commerciale - Partage dans les Mêmes Conditions 4.0 International. 


\title{
Les Kinsey des deux Allemagnes : recherche empirique en sexologie, enquêtes sur la sexualité et normalisation de la sexualité de la jeunesse pendant la guerre froide ${ }^{1}$
}

\author{
Lutz SAUERTEIG
}

\section{Introduction}

Le présent article interroge comment, à la suite de la publication des rapports Kinsey, la sexologie, en Allemagne de l'Est et de l'Ouest, se tourna vers la recherche empirique. Nous analysons plus précisément le contexte politique et culturel de la guerre froide dans lequel les sexologues et les chercheurs en sciences sociales d'Allemagne de l'Ouest et de l'Est menèrent des enquêtes sur les comportements sexuels des jeunes et sur leurs valeurs morales. Après avoir présenté notre approche théorique, nous examinons la (ré)institutionnalisation de la sexologie en Allemagne de l'Ouest et de l'Est ainsi que les controverses qui émergèrent au sujet des rapports Kinsey dans les années 1960. Dans une troisième partie, nous analysons le virage vers la recherche empirique en sexologie qui se produisit des deux côtés du rideau de fer. L'avant-dernière partie présente les résultats des enquêtes sur la sexualité menées en Allemagne de l'Est et de l'Ouest pendant les années 1960 et 1970 . Nous soutenons l'idée que les données statistiques produites par ces enquêtes sur la sexualité ont non seulement créé une réalité historique de la vision de la sexualité des jeunes et de leurs comportements sexuels, mais qu'elles ont également façonné de nouveaux critères de «normalité ». Ces données statistiques deviennent, en effet, des événements discursifs : l'on y fait souvent référence, elles sont vulgarisées et présentées sous la forme de sondage du lectorat par les grands médias et elles sont représentées

${ }^{1}$ Nous avons présenté des versions antérieures de cet article à des conférences tenues à l'Université libre de Bruxelles, à l'Exeter University, et notre réflexion a été enrichie par les questions et des discussions que nous y avons entendues. Nous remercions également la Dr B. Cross et les relecteurs anonymes pour leurs commentaires et leurs suggestions. 
sous forme de graphiques ${ }^{2}$. De tels événements discursifs incitent alors les jeunes à rapporter leurs propres jugements moraux et leurs propres comportements sexuels à la moyenne statistique de leur classe d'âge et, par conséquent, façonnent chez eux la perception de ce qui est acceptable dans un contexte culturel donné.

Le concept de normalisation a bien montré que la publication de données issues des enquêtes sur la sexualité augmente la flexibilité de l'acceptabilité en ce qui concerne la morale et les comportements sexuels. À partir des concepts foucaldiens de biopouvoir et de normalisation, Jürgen Link interprète l'interdépendance entre les données obtenues par les sondages et leurs répondant $\cdot \mathrm{e} \cdot \mathrm{s}$ comme relevant d'un processus de normalisation. Ce processus se produit à travers la production culturelle et la reproduction de normalités qui deviennent, alors, des conceptions partagées dans la société ${ }^{3}$. Au XIX ${ }^{\mathrm{e}}$ siècle et jusqu'à la moitié du $\mathrm{XX}^{\mathrm{e}}$ siècle, certains comportements ou caractéristiques sexuels étaient disqualifiés comme pervers ou pathologiques, dans le cadre de ce que Link appelle une stratégie « protonormaliste $»^{4}$. Cette stratégie sépare par des limites solides et rigides le «normal » du « pervers ». Elle exige, de la part de la société, des mécanismes de réglementation et de répression qui permettent à une subjectivité hétéronome de « normaliser » avec force les individus. Bien qu'elle parvienne à maîtriser les déviations de la normalité, la stratégie protonormaliste empêche, selon Link, « la dynamique moderne de la croissance $»$ et n'accepte pas les transgressions ${ }^{5}$.

La " stratégie normaliste flexible » qui prévaut depuis environ la deuxième partie du $\mathrm{XX}^{\mathrm{e}}$ siècle a quant à elle transformé, au moyen de données statistiques, les comportements et les identités sexuels en deux catégories : d'une part ceux qui relèvent du commun, ou du «normal », au sens statistique de moyen, et d'autre part ceux qui sont rares. Ainsi, les données des enquêtes sur la sexualité ont montré que quelque chose comme la masturbation ou l'homosexualité - qui était considéré comme pathologique dans le cadre de la stratégie protonormaliste était, en fait, pratiqué par un nombre

${ }^{2}$ Sur ces représentations graphiques, voir. S. NIKOLOW, «Imaginäre Gemeinschaften. Statistische Bilder der Bevölkerung ", in M. Hessler (dir.), Konstruierte Sichtbarkeiten. Wissenschafts- und Technikbilder seit der Frühen Neuzeit, Munich, Fink, 2006, p. 263-278 ; J. LINK, "The normalistic subject and its curves: on the symbolic visualization of orienteering data » [trad. de M. M. Hall], Cultural Critique, 2004, n 57, p. 47-67 ; U. Gerhard, J. Link et E. Schulte-Holtey (dir.), Infografiken, Medien, Normalisierung. Zur Kartografie politischsozialer Landschaften, Heidelberg, Synchron, 2001.

3 J. Link, Versuch über den Normalismus. Wie Normalität produziert wird, Göttingen, Vandenhoeck et Ruprecht, 1997, $4^{\mathrm{e}}$ éd., 2009, $1^{\text {re }}$ partie et p. 73-80 ; J. LinK, « From the "power of the norm" to "flexible normalism": considerations after Foucault » [trad. de M. M. Hall], Cultural Critique, 2004, $\mathrm{n}^{\circ}$ 57, p. 14-32; A. B. BüHRMANN, « Die gesellschaftlichen Konsequenzen der Wissensproduktion. Zum Verhältnis von (Sexual-)Wissenschaften und gesellschaftlichen Normalisierungsmechanismen ", in U. Ferdinand, A. Pretzel et A. SeeK (dir.), Verqueere Wissenschaft? Zum Verhältnis von Sexualwissenschaft und Sexualreformbewegung in Geschichte und Gegenwart, Münster, Lit, 1998, p. 213-228. Sur les différences linguistiques entre « norme », « normal », «normalisation », « normatif » et « standardisation » en français, anglais et allemand, voir J. Link, « From the "power of the norm" to "flexible normalism"», op. cit., p. 14-19.

${ }^{4}$ J. Link, ibid., p. 27 ; J. Link, Versuch über den Normalismus, op. cit., p. 51-62; A. B. BÜHRMANN, op. cit., p. 217-219.

5 J. LinK, "From the "power of the norm" to "flexible normalism" », op. cit., p. 28. 
significatif d'individus et, par conséquent, devait entrer dans la catégorie normale, au sens statistique. Alors, une nouvelle forme de "sexualité normale »- au sens de caractéristique pour la moyenne - est apparue. À présent, des limites flexibles encadrent cette « sexualité normale » qui créent de vastes « zones de tolérance et de transition » qui admettent mieux les transgressions et gèrent mieux les écarts, mais qui portent en elles un risque accru de « dissolution des limites $»^{6}$. Ces nouvelles normalités flexibles n'existent que « dans les sociétés qui reposent sur l'analyse de données », des sociétés qui « constamment, régulièrement, avec exhaustivité, de façon institutionnalisée acceptent, avec transparence, de se montrer telles qu'elles sont, à l'aide de la statistique ${ }^{7}$.

Les données publiées à partir des études sur la sexualité permettent aux répondant $\cdot \mathrm{e} \cdot \mathrm{s}$ et à leurs pairs de comparer et de classer leurs propres comportements, pratiques et croyances par rapport à ce qui leur est présenté soit comme dans la moyenne et, en ce sens, comme normal, soit comme peu fréquent mais pas pour autant pathologique. Le normalisme flexible demande en réalité aux individus de se «normaliser » eux-mêmes. Il leur faut trouver, à travers un processus d' « auto-adaptation dynamique » et de « choix tactiques », leur place dans le spectre du normal qui va d'un milieu moyen jusqu'aux « zones de tolérance » et à « la limite de la normalité »". L'autonormalisation demande un entraînement, par exemple, comme Link le suggérait, à l'aide de " programmes d'entraînement psychothérapeutique au sens le plus large » ou, ajoutons encore, à l'aide de matériaux d'éducation sexuelle ${ }^{9}$. Les limites de la normalité peuvent s'avérer plus attirantes quand ce qui est dans la moyenne semble ennuyeux. De même, les données concernant des performances élevées, par exemple sur la fréquence des orgasmes, peuvent être perçues comme un défi à relever ${ }^{10}$. La dissolution des limites, auparavant strictes, de ce qui est sexuellement acceptable nécessite également qu'un nouveau code éthique soit élaboré. Selon les termes de ce nouveau code, des partenaires responsables et égaux·ales devront négocier pour parvenir à s'entendre sur la nature de leurs relations érotiques et sexuelles.

\section{Institutionnalisation de la sexologie en Allemagne de l'Ouest et de l'Est}

Bien que la recherche en sexologie ait un peu continué sous le Troisième Reich, toutefois, sous l'angle de l'eugénisme et du racisme, l'arrivée au pouvoir des nazis en $1933^{11}$ a mis un coup d'arrêt brutal à la prolifération qu'elle avait connue sous la

${ }^{6}$ Ibid.; A. B. BüHRMANN, op. cit., p. 221-225.

7 J. Link, "From the "power of the norm" to "flexible normalism" », op. cit., p. 18 ; J. LinK, «On the contribution of normalism to modernity and postmodernity » [trad. de M. M. Hall], Cultural Critique, 2004, n 57, p. 33-46, p. 35. Selon J. Link, afin de rendre une société statistiquement transparente, il convient de publier les données collectées par la surveillance d'État (il mentionne la Stasi, la police secrète de la RDA) et celles recueillies par les sondages d'opinion.

8 J. Link, "From the "power of the norm" to "flexible normalism" », op. cit., p. 29.

${ }^{9}$ Ibid.

${ }^{10}$ J. LiNK, « On the contribution of normalism to modernity and postmodernity », op. cit., p. 42 ; J. Link, Versuch über den Normalismus, op. cit., p. 74.

${ }^{11}$ A. Grossmann, Reforming Sex: The German Movement for Birth Control and Abortion reform, 1920-1950, New York et Oxford, Oxford University Press, 1995 ; K. von Soden, Die Sexualberatungsstellen der Weimarer Republik, 1919-1933, Berlin, Hentrich, 1988; V. Sigusch, Geschichte der Sexualwissenschaft, Francfort et New York, Campus, 2008, partie B. 
République de Weimar. Après la fin de la guerre, des sexologues, dont certains avaient participé au régime nazi avec des degrés d'implication variables, essayent de réétablir et d'institutionnaliser la recherche sur la sexualité, en Allemagne de l'Est comme de l'Ouest. Ainsi, en avril 1949, Hans Giese fonde un Institut pour la recherche sur la sexualité (Institut für Sexualforschung) à son domicile de Kronberg, aux environs de Francfort. À la suite de plaintes du voisinage, il doit rapidement déplacer cet institut dans l'appartement de ses parents, en ville, puis, en 1959, à Hambourg, où il s'associe à l'hôpital universitaire $^{12}$. H. Giese, qui est docteur en philologie germanique et en médecine, devient le père fondateur d'une nouvelle génération de sexologues allemands. Lui et son mentor, le psychiatre Hans Bürger-Prinz, un ancien membre du Parti national-socialiste et d'autres organisations nazies, qui avait participé aux campagnes de stérilisation et d'hygiène raciale, constituent les figures dominantes de la sexologie ouest-allemande jusqu'aux années 1970. C'est ainsi qu'est créée, sous l'impulsion de H. Giese, la Société allemande de sexologie (Deutsche Gesellschaft für Sexualforschung, DGfS), en avril 1950, société dont H. Bürger-Prinz devient le premier président ${ }^{13}$.

En Allemagne de l'Est, les tentatives pour le réétablissement et l'institutionnalisation de la sexologie rencontrent davantage d'obstacles, l'État socialiste y faisant notamment obstruction ${ }^{14}$. Il s'avère, ainsi, impossible de republier les manuels d'éducation sexuelle des années 1920 du médecin socialiste Max Hodann. Dans ces mêmes années 1920, l'URSS avait déjà désavoué l'œuvre de Wilhelm Reich qui constituait, à ses yeux, une attaque contre le marxisme-léninisme. De plus, la psychanalyse y est discréditée. Les psychologues et les spécialistes de l'éducation d'Allemagne de l'Est doivent adopter le jargon du matérialisme et suivre les approches de chercheurs soviétiques, comme la théorie de la réflexologie du physiologiste Ivan Pavlov ou le matérialisme éducatif d'Anton Makarenko. A. Makarenko - qui devient, en RDA, la référence dogmatique absolue en matière de didactique - considère la sexualité, et le fait d'en parler, comme une menace majeure pour la société. Aussi rejette-t-il toute forme d'éducation sexuelle ${ }^{15}$.

Ce n'est qu'après la mort de Staline en 1953, avec la période de dégel qui s'ensuit à partir de 1956, que le régime d'Allemagne de l'Est, sous la direction de Walter Ulbricht, change de position sur l'éducation sexuelle, ce qui modifie,

${ }^{12}$ V. Sigusch, ibid., ch. 18.

${ }^{13}$ Ibid., ch. 19 ; M. LieBEKNECHT, « Sexualität als Gegenstand der Zeitgeschichtsforschung und der Sexualwissenschaft in der frühen Bundesrepublik », Zeitschrift für Sexualforschung [Z. Sexualforsch.], 28, 2015, p. 132-148 ; S. SteinBaCher, Wie der Sex nach Deutschland kam. Der Kampf um Sittlichkeit und Anstand in der frühen Bundesrepublik, Munich, Siedler, 2011, p. 219-220 ; P. von RöNN, « Die Homosexualitätsentwürfe von Hans Giese und der lange Schatten von Hans Bürger-Prinz », Z. Sexualforsch., 13, 2000, p. 277-310 ; S. BECKER, « Bemerkungen zur Debatte über Bürger-Prinz », Z. Sexualforsch., 4, 1991, p. 265-270.

${ }^{14}$ G. Grau, « Sexualwissenschaft in der DDR: Ein Resümee », in V. Sigusch, Geschichte der Sexualwissenschaft, Francfort et New York, Campus, 2008, p. 487-509 ; P. G. Hesse, « Die Anfänge der Sexuologie in der DDR », in J. S. Hohmann (dir.), Sexuologie in der DDR, Berlin, Dietz, 1991, p. 51-61, p. 55-56.

${ }^{15}$ M. Fenemore, "The growing pains of sex education in the German Democratic Republic (GDR), 1945-1969 », in L. SAuerteig et R. Davidson (dir.), Shaping Sexual Knowledge: A Cultural History of Sex Education in Twentieth Century Europe, Londres et New York, Routledge, 2009, p. 74. 
également, l'attitude à l'égard de la recherche sexologique ${ }^{16}$. Cependant, la $5^{\text {e }}$ conférence du Parti socialiste unifié (Sozialistische Einheitspartei Deutschlands, SED) en juillet 1958 sonne déjà la fin de cette courte période de dégel. Ulbricht y proclame, avec les Dix commandements de l'éthique et de la morale socialiste, une nouvelle forme de moralité sexuelle socialiste qui devient la nouvelle référence morale en RDA. Le neuvième de ces commandements exige de chaque citoyen ne de mener une vie " propre et saine » et de respecter sa famille ${ }^{17}$. Pour l'État socialiste, les relations sexuelles ne relèvent pas de la sphère privée. Au contraire, il insiste sur leur dimension sociale. Celle-ci justifie l'intervention de l'État, par exemple dans son combat contre la prostitution ou dans son interdiction de la pornographie, deux phénomènes qui sont considérés comme étant intrinsèquement liés au capitalisme ${ }^{18}$.

Dans le cadre dogmatique de cette nouvelle morale sexuelle socialiste, la sexologie est-allemande demeure une science normative jusque fin des années 1960. Les sexologues et les pédagogues sexuel·le·s condamnent toute forme de relations sexuelles en dehors du mariage. L'un des éducateurs sexuels les plus importants de l'époque, le professeur d'hygiène sociale Rudolf Neubert, met les jeunes gens en garde contre des pratiques déplorables telles que les caresses érotiques. Lui et d'autres éducateurs sexuels et sexologues de RDA demandent aux jeunes gens de s'abstenir d'avoir des relations sexuelles avant le mariage et de limiter leur vie sexuelle au cadre de la famille socialiste ${ }^{19}$.

La morale sexuelle socialiste des années 1950 et du début des années 1960 est donc très proche de la morale chrétienne en vigueur en Allemagne de l'Ouest. Son insistance sur le mariage, sur les valeurs familiales traditionnelles et les normes hétérosexuelles fondées sur le genre est manifeste dans les documents d'éducation

${ }^{16}$ Ibid., p. 75-78 ; D. Herzog, Sex After Fascism: Memory and Morality in TwentiethCentury Germany, Princeton et Oxford, Princeton University Press, 2005, p. 192-195.

${ }_{17}$ Protokoll der Verhandlungen des V. Parteitages der Sozialistischen Einheitspartei Deutschlands: 10. bis 16. Juli 1958 in der Werner-Seelbinder-Halle zu Berlin, 2 vols, Berlin [East], Dietz, 1959, vol. 1, p. 160 ; W. UlBRICHT, « Sozialistische Ethik und Moral», Elternhaus und Schule, 7/11, 1958, p. 15 ; voir J. McLellan, Love in the Time of Communism: Intimacy and Sexuality in the GDR, Cambridge et New York, Cambridge University Press, 2011, p. 7 ; U. G. PoIger, Jazz, Rock, and Rebels: Cold War Politics and American Culture in a Divided Germany, Berkeley, Los Angeles et London, University of California Press, 2000, p. 194 ; M. Fenemore, Sex, Thugs and Rock 'n'Roll: Teenage Rebels in Cold-War East Germany, New York et Oxford, Berghahn Books, 2009, ch. 2.

${ }^{18}$ Voir F. Steger et M. Schochow, Disziplinierung durch Medizin. Die geschlossene Venerologische Station in der Poliklinik Mitte in Halle (Saale) 1961 bis 1982, Halle, Mitteldeutscher Verlag, 2014 ; J. McLellan, op. cit., p. 98-102, 174-175 ; M. Fenemore, Sex, Thugs and Rock 'n' Roll, op. cit., p. 23, 35 ; voir D. Herzog, op. cit., p. 202, 205, 219.

${ }_{19}$ R. NEUBERT, Die Geschlechterfrage. Ein Buch für junge Menschen, Rudolstadt, Greifenverlag, $4^{e}$ éd. révisée, 1956, p. 151-152 ; H.-J. Hoffmann et P. G. KLEMm, Ein offenes Wort. Ein Buch über die Liebe, Berlin[-est], Neues Leben, 1956, p. 112 ; voir D. HerzoG, op. cit., p. 198-200. 
sexuelle de l'époque ${ }^{20}$. Par exemple, le docteur Christoph Vollmer, à partir de 1962 , dans ses articles hebdomadaires pour Bravo - le journal pour adolescent·e $\cdot s$ le plus célèbre d'Allemagne de l'Ouest - demande à son jeune lectorat de patienter jusqu'au mariage pour avoir des relations sexuelles ${ }^{21}$. De même, le Centre fédéral pour l'éducation sanitaire (Bundeszentrale für gesundheitliche Aufklärung, BZgA), dans une brochure publiée en 1967 pour accompagner l'éducation sexuelle à l'école, refuse de mentionner la pilule contraceptive au nom d'un scrupule moral, de crainte que cela n’encourage la jeunesse à avoir des rapports sexuels préconjugaux ${ }^{22}$.

\section{L'après-Kinsey : la bataille pour la sexologie empirique et le normalisme flexible}

À l'inverse de ce qui se passe aux États-Unis et au Royaume-Uni, il n'y a pas de tradition de recherche par enquêtes empiriques en Allemagne après la guerre. Ce type de recherche constitue même une nouveauté pour la plupart des chercheur·euse·s allemand·e's en sciences humaines, et en particulier pour les sexologues, avec leur formation de type médical ${ }^{23}$. En dehors du cas particulier de la sexologie allemande, à l'Est comme à l'Ouest, la recherche empirique sur la sexualité explose, en revanche, dans la sphère publique juste après la fin de la Seconde Guerre mondiale avec les recherches pionnières d'Alfred Kinsey sur le Comportement sexuel de l'homme en 1948 et sur le Comportement sexuel de la femme en $1953^{24}$. Les traductions allemandes de ces deux rapports suivent rapidement, respectivement en 1955 et en

${ }^{20}$ L. SAuerteIG, « Junge oder Mädchen - Frau oder Mann? Der heterosexuelle Körper und die Herstellung visueller Selbstverständlichkeiten in der Sexualaufklärung im 20. Jahrhundert », WerkstattGeschichte, 16/47, 2007, p. 40-60 ; L. SAUERTEIG, " Representations of pregnancy and childbirth in (West) German sex education books, 1900s-1970s », in L. Sauerteig et R. Davidson (dir.), Shaping Sexual Knowledge: A Cultural History of Sex Education in Twentieth Century Europe, Londres et New York, Routledge, 2009, p. 138-140.

${ }^{21}$ Ch. Vollmer, "Verliebt, geliebt und liebenswert ", Bravo, 1964, n 26, p. 43. Voir L. Sauerteig, « Die Herstellung des sexuellen und erotischen Körpers in der westdeutschen Jugendzeitschrift BRAVO in den 1960er und 1970er Jahren ", Medizinhistorisches Journal, 42, 2007, p. 149-151.

${ }^{22}$ Bundearchiv, B304/3125, BZgA, pour le Bundesministeriums für Gesundheitswesen, Beiträge zur Geschlechtserziehung, partie I : Biologie der Fortpflanzung, Cologne, 1967, p. 7.

${ }^{23}$ Ch. Reinecke, " Statistiken der Liebe oder: Dr. Kinsey fragt die Frauen. Umfrageforschung und ihre mediale Vermarktung in transnationaler Perspektive », Comparativ, 21/4, 2011, p. 30-31, 39-40 ; Ch. WeISCHER, Das Unternehmen „Empirische Sozialforschung “. Strukturen, Praktiken und Leitbilder der Sozialforschung in der Bundesrepublik Deutschland, Munich, Oldenbourg, 2004 ; H. Giese et G. Schmidt, Studentensexualität. Verhalten und Einstellung. Eine Umfrage an 12 westdeutschen Universitäten, Reinbek, Rowohlt, 1968, p. 11.

${ }^{24}$ Voir S. E. Igo, The Averaged American: Surveys, Citizens, and the Making of a Mass Public, Cambridge, Mass., et Londres, Harvard University Press, 2007, p. 6-22 ; L. STANLey, Sex Surveyed, 1949-1994: From Mass-Observation's "Little Kinsey" to the national survey and the Hite Reports, Londres, Taylor et Francis, 1995 ; J. M. Converse, Survey Research in the United States: Roots and Emergence 1890-1960, Berkeley et Londres, University of California Press, 1987. 
$1954^{25}$. On peut les considérer comme les premiers documents qui témoigneraient d'un normalisme flexible en matière de recherche sexologique ${ }^{26}$.

Inspiré par le premier rapport Kinsey, un hebdomadaire illustré d'Allemagne de l'Ouest, Wochenend («Week-end»), commande, en 1949, à l'institut de sondage Allensbach une enquête sur le comportement sexuel des Allemand·e's de l'Ouest ${ }^{27}$. Wochenend publie les résultats de cette étude sous le titre « Le pouvoir élémentaire de l'amour à notre époque » en série, entre novembre 1949 et février 1950. De plus, peu avant la parution des traductions allemandes des rapports Kinsey, le sociologue Ludwig von Friedeburg publie une analyse plus détaillée des données de l'enquête ${ }^{28}$. Le magazine avance que c'est la « valeur probante » sans précédent de ces données statistiques transversales sur les mentalités et les comportements sexuels des Allemand·e's de l'Ouest qui « balayeraient un grand nombre d'idées fausses » sur la sexualité ${ }^{29}$. Tout en actant la disparition en cours des limites fixes qui déterminent ce qui est considéré comme sexuellement acceptable, le magazine soutient que l'étude montrerait que l'Allemagne de l'Ouest n'est pas dans un état de " décadence morale ${ }^{30}$, contrairement à l'opinion de certain·e's commentateur'rice's de l'époque. En Allemagne de l'Ouest, cette étude constitue la première recherche sur la sexualité basée sur des données statistiques transversales collectées par une enquête plutôt qu'à partir de cas recueillis par des médecins, des psychiatres ou des psychanalystes dans leurs cabinets ou dans leurs hôpitaux. Aussi cette enquête peut-elle être considérée comme une étape importante vers une stratégie normaliste flexible en Allemagne de l'Ouest.

Cependant, malgré l'intérêt significatif que suscite, au début des années 1950, cette nouvelle forme empirique de recherche sexologique dans le grand public, et bien que la Société allemande de sexologie ait publié les analyses sociologiques de von Friedeburg, cette étude passe largement inaperçue de la majorité des sexologues et elle demeure une exception ${ }^{31}$. La sexologie ouest- - et est- - allemande continue à être dominée par des chercheur·se·s avec une formation médicale, partisan·e·s de la stratégie protonormaliste, et non pas par des chercheur·e·s en sciences sociales.

${ }^{25}$ A. C. KInsey et al., Das sexuelle Verhalten der Frau, [Sexual Behavior in the Human Female, Philadelphie et Londres, Saunders, 1953], [trad. de M. Baacke et al.], Berlin et Francfort, Fischer, 1954, réimpr. 1967 ; A. C. Kinsey, W. B. Pomeroy et C. E. Martin, Das sexuelle Verhalten des Mannes, [Sexual Behaviour in the Human Male, Philadelphie, Saunders, 1948], [trad. de M. von Eckardt-Jaffé, M. Baacke et W. Seemann], Berlin et Francfort, Fischer, 1955, réimpression 1967.

26 J. Link, Versuch über den Normalismus, op. cit., p. 74.

27 « Intimsphäre », Jahrbuch der öffentlichen Meinung, 3/1958-1964, 1965, p. 587-593 ; voir S. STEINBACHER, op. cit., p. 156-163.

28 "Urmacht Liebe in unserer Zeit. Die große deutsche Sexual-Analyse », Wochenend, 10 novembre 1949-16 février 1950 ; F. vON FrIedeburg, Die Umfrage in der Intimsphäre, Stuttgart, Enke, 1953 ; voir Ch. ReINECKE, op. cit., p. 39-43.

${ }^{29}$ H. W., «Urmacht Liebe in unserer Zeit », Wochenend, n 45, 10 novembre 1949, p. 1.

${ }^{30}$ Ibid.

31 M. Dannecker, « Die verspätete Empirie. Anmerkungen zu den Anfängen der Deutschen Gesellschaft für Sexualforschung », Z. Sexualforsch., 14, 2001, p. 167. 
La controverse qui suit la publication de la traduction allemande des rapports Kinsey, les critiques sévères qui sont portées sur leur méthodologie et sur leurs conséquences quant à la morale sexuelle montrent que le passage d'une stratégie protonormaliste à une stratégie normaliste flexible ne se fait pas sans rencontrer de résistance ${ }^{32}$. Cette controverse constitue un sérieux avertissement pour quiconque aurait envisagé d'entreprendre des recherches empiriques en sexologie à cette époque. En effet, tandis que les sexologues sont en train de parvenir à faire de la sexologie une science respectable en Allemagne de l'Ouest, l'opinion publique s'inquiète de plus en plus de la croissance de l'industrie pornographique, de l'abondance des images érotiques et sexualisées dans les magazines, les publicités et les films qui semblent remettre en cause les normes sexuelles traditionnelles ${ }^{33}$. Ainsi, quand deux magazines populaires (Quick et Stern) approchent H. Giese en 1966 pour publier les résultats de la recherche empirique qu'il a menée avec Gunter Schmidt sur la sexualité des étudiant $\cdot \mathrm{e} \cdot \mathrm{s}^{34}$, celui-ci se montre réticent en raison des attaques que Kinsey a subies suite à la vulgarisation de sa recherche sexologique. Il estime, en effet, que les résultats de recherches empiriques en matière de sexologie ne sont pas destinés à être communiqués au grand public et doivent être réservés aux « experts ${ }^{35}$. Le périodique Der Spiegel commente tout de même, de façon assez détaillée, les résultats de sa recherche ${ }^{36}$.

Parmi les critiques allemands d'A. Kinsey se trouve l'influent Helmut Schelsky. Sociologue conservateur, ami de H. Bürger-Prinz, H. Schelsky a rejoint la SA en 1932 et adhéré au Parti nazi en 1937. Il critique chez A. Kinsey son explication biologique du comportement sexuel et il avance l'idée que la sexualité ainsi que les comportements sexuels sont avant tout déterminés par des facteurs socioculturels ${ }^{37}$. L'intérêt immense que l'opinion publique porte aux rapports l'inquiète particulièrement. Selon lui, la vulgarisation de l'information portant sur les comportements et les expériences sexuelles - et tout particulièrement sur l'homosexualité - détruit les façons traditionnelles de penser la sexualité et les valeurs morales. Il estime que cette vulgarisation est dangereuse en ce qu'elle créerait de nouvelles normes et qu'elle

${ }^{32}$ Ch. Reinecke, op. cit. ; S. Steinbacher, op. cit., p. 152-156, 166-203 ; D. Herzog, "The reception of the Kinsey reports in Europe », Sexuality and Culture, 10/1, 2006, p. 39-48 ; G. SCHмIDT, « Kinsey's unspoken truth: on how the Kinsey reports were received in West Germany », Sexualities, 1, 1998, p. 100-103; M. DANNeCKER, "Kann empirische Sexualforschung kritisch sein? Zum Andenken an Alfred C. Kinsey », Z. Sexualforsch., 2, 1989, p. 207-215 ; R. ReIche, « Die Aufnahme der Kinsey-Berichte», Vorgänge, 5, 1966, p. 364-372.

33 S. Steinbacher, op. cit., p. 218-219, 267-324 ; E. D. Heineman, Before Porn was Legal: The Erotica Empire of Beate Uhse, Chicago, Ill., University of Chicago Press, 2011 ; M. LiebeKneCht, " „Wir ertrinken in der Sex-Welle“. Hans Giese und der öffentliche Sexualitätsdiskurs in den sechziger Jahren », Zeitgeschichte in Hamburg, 2016, p. 52-67.

${ }^{34}$ H. Giese et G. SCHMidT, op. cit.

${ }^{35}$ M. Liebeknecht, " „Wir ertrinken in der Sex-Welle“" S. STEINBACHER, op. cit., p. 218.

36 « Sex-Report: Nur wenig », Der Spiegel, 26 août 1968, n 35, p. 46-50.

${ }^{37}$ H. SCHELSKy, Soziologie der Sexualität. Über die Beziehungen zwischen Geschlecht, Moral und Gesellschaft, Hambourg, Rowohlt TB, 1955, p. 11-14, 49-50, 102. 
pourrait donc menacer le socle culturel d'une nation ${ }^{38}$. Autrement dit, H. Schelsky craint la disparition des limites rigides qui entourent la moralité et la sexualité, leur assouplissement. Il estime, en effet, que les rapports Kinsey ont fait « de la biologie et de la statistique des sciences normatives $»^{39}$.

D'autres commentateur'ice's conservateur'rice's, comme Walter Petwaidic - un ancien bureaucrate nazi qui, sous le Troisième Reich, a travaillé dans le service de presse et communication des Affaires étrangères -, avertissent l'hebdomadaire Die Zeit en novembre 1953 que cette nouvelle " méthode américaine pour déterminer une moyenne humaine » pourrait mener à une fragmentation accrue de la société. Accusant Kinsey de «matérialisme sexuel», W. Petwaidic craint que de telles enquêtes débouchent sur une publicisation à outrance de la vie privée. Il pense également que la méthode utilisée par ces enquêtes sur la sexualité est fallacieuse, car « il va de soi que le simple fait de bien vouloir répondre à des questions aussi intimes que celles posées par Kinsey et ses collaborateurs témoigne déjà d'un certain niveau d'anormalité ». Aussi, selon lui, ces enquêtes ne parviennent pas à saisir une image "vraie » de l'activité sexuelle humaine ${ }^{40}$.

À l'autre bout du spectre politique, le sociologue Reimut Reiche redoute, lui aussi, les effets d'un normalisme flexible. Figure militante de premier plan du mouvement étudiant de 1968, R. Reiche craint que la vulgarisation par les médias généralistes de données statistiques issues des enquêtes sur la sexualité ait un effet normalisant, qu'elles créent, pour les comportements sexuels, des injonctions " plus douces » et "déguisées » de conformité aux moyennes statistiques ${ }^{41}$. Günter Amendt, un autre jeune sociologue, lui aussi militant du mouvement étudiant, critique également la recherche sexologique empirique. Il déclare, avec provocation, en 1970 que « le fait de regarder des données transversales [Querschnitt] provoque la paraplégie des fantasmes chez les chercheurs empiriques ». Selon lui, la recherche empirique sexologique réduirait l'intimité des expériences sexuelles à des pratiques sexuelles quantifiables et à des positions et ne saurait participer au développement d'une position critique contre le capitalisme : «Le fantasme de la recherche empirique correspond au fantasme menotté du Capital. $»^{42}$ La résistance contre une stratégie normaliste flexible n'est donc pas le seul fait de l'arrière-garde des sociologues d'Allemagne de l'Ouest, retranché $\cdot \mathrm{e} \cdot \mathrm{s}$ derrière leur approche protonormaliste, plus proches du conservatisme politique. Le scepticisme, au moins au départ, provient aussi de sociologues plus jeunes, issu $\cdot e \cdot s$ de la nouvelle gauche.

${ }_{38}$ Ibid., p. 7-8, 48-52, 57 ; sur l'homosexualité, p. 75-87 ; voir S. STEINBACHER, op. cit., p. 221-237.

${ }^{39}$ H. SCHELSKY, op. cit., p. 52.

${ }^{40}$ W. Fredericia [Walter Petwaidic], « Kinsey über die amerikanische Frau: Die Vivisektion des Menschen mit dem Messer des modernen Zoologen », Die Zeit, 19 novembre $1953, n^{\circ} 47$.

${ }^{41}$ R. Reiche, « Kritik der gegenwärtigen Sexualwissenschaft», in G. Schmidt, V. Sigusch et E. Schorsch (dir.), Tendenzen der Sexualforschung, Stuttgart, Enke, 1970, p. 5-6.

${ }^{42}$ En allemand : «Die Phantasie der Empirie ist die gefesselte des Kapitals. » G. AmEndT, «Empirie, Emanzipation und Sexualforschung », in G. SCHMidt, V. Sigusch et E. Schorsch (dir.), Tendenzen der Sexualforschung, Stuttgart, Enke, 1970, p. 18. 
En Allemagne de 1'Est, des reproches similaires - et donc des craintes analogues quant aux conséquences d'une stratégie normaliste flexible - sont exprimés par les sexologues où, là aussi, la publication des rapports Kinsey est condamnée. Rudolf Neubert estime, dans son livre de conseils sur le mariage et la sexualité de 1957, que le lectorat est-allemand ne comprendrait pas que le rapport n'est valable que pour l'Amérique du Nord. R. Neubert, qui a connu des difficultés dans sa carrière en RDA en raison de son passé en tant que membre du Parti nazi, semble rejeter la recherche sexologique empirique dans son ensemble. Il accuse A. Kinsey de seulement décrire des comportements sexuels sans fournir à son lectorat des «normes » et des « conseils » pour leur propre vie sexuelle, ce qui constitue précisément l'objet d'une stratégie normaliste flexible. Pour lui, des statistiques en elles-mêmes ne sauraient fournir des normes valides - quand bien même ces statistiques auraient été basées sur un échantillon significatif, ce dont il doute par ailleurs pour les rapports Kinsey ${ }^{43}$. Il s'inquiète aussi du fait que l'approche empirique et statistique de Kinsey et le débat public sur les pratiques et les techniques sexuelles détruiraient le caractère individuel et intime des expériences sexuelles ${ }^{44}$. De plus, il estime que la recherche statistique est incapable de résoudre les nouveaux défis posés par les rapports de genre qui devraient être abordés à l'intérieur du cadre moral édifié par la société socialiste et via un « long processus éducatif social ${ }^{45}$. Heinz Grassel, un psychopédagogue de l'Université de Rostock, craint, de même, qu'une recherche sexologique exclusivement empirique " transforme la fréquence statistique en normes » en matière de comportements sexuels ${ }^{46}$. Tout comme H. Schelksky, H. Grassel accuse A. Kinsey d'épouser une approche behaviouriste de la sexualité qui en négligerait les aspects psychologiques. Il est convaincu, comme nombre de commentateur $\cdot$ rice's ouest-allemand $\cdot e \cdot s$, que les résultats des recherches sexologiques ne doivent pas, en général, être rendus publics et doivent être réservés au cercle étroit des spécialistes ${ }^{47}$.

À l'exception de quelques enquêtes de moindre échelle, la sexologie est-allemande demeure donc fondamentalement protonormaliste jusqu'au début des années 1960, soutenant le dogme de moralité et de la famille socialiste. Les autorités et les idéologues de la RDA regardent généralement les sciences sociales empiriques avec suspicion. Ils en rejettent les méthodes de recherche, comme les sondages d'opinion, les enquêtes. Ils les perçoivent comme des « méthodes de production de connaissances bourgeoises et pseudoscientifiques » qui ne servent qu' « à masquer [et] à déformer la réalité » et qui ne contribueraient pas à la construction d'une nouvelle société socialiste ${ }^{48}$. Plutôt que

${ }^{43}$ R. NeUbert, Das neue Ehebuch. Die Ehe als Aufgabe der Gegenwart und Zukunft, Rudolstadt, Greifenverlag, 1957, 20éd. révisée, 1972, p. 166-167.

${ }^{44}$ Ibid., p. 167.

${ }^{45}$ Ibid., p. 168-169, citation p. 168.

${ }^{46}$ H. Grassel, «Zur Problematik der Methoden der Sexualforschung », Wissenschaftliche Zeitschrift der Universität Rostock: Gesellschafts- und sprachwissenschaftliche Reihe, 16, 1967, p. 381.

${ }^{47}$ Ibid.; H. Grassel, Jugend, Sexualität, Erziehung. Zur psychologischen Problematik der Geschlechtserziehung, Berlin[-est], Staatsverlag der DDR, 1967, p. 48, 50.

${ }^{48}$ W. FRIEDRICH, « DDR-Jugendforschung - zwischen wissenschaftlichem Anspruch und politischer Bevormundung », in W. FRIEDRICH et H. GRIESE (dir.), Jugend und Jugendforschung 
d'enquêter sur les attitudes et les comportements de la jeunesse et donc d'adopter une stratégie normaliste flexible, les chercheur'se's en sciences humaines d'Allemagne de l'Est doivent propager et faire appliquer la politique socialiste officielle pour la jeunesse et la moralité socialiste et, donc, protéger les limites rigides de la moralité socialiste dans le cadre d'une hégémonie protonormaliste.

Parce que la sexualité est principalement abordée en termes de moralité normative jusqu'aux années 1960, les commentateur·ice's et les scientifiques partisan·e·s de la stratégie protonormaliste en Allemagne de l'Ouest et de l'Est craignent que la communication au grand public des résultats d'enquêtes sexologiques serve à appuyer des demandes de changement en matière de moralité sexuelle ${ }^{49}$. De fait, Kinsey a bien demandé que la loi soit changée quand elle allait à l'encontre des pratiques de nombreux Américains, surtout en ce qui concerne les actes homosexuels qui, selon les enquêtes, sont le fait d'un quart à un tiers de la population ${ }^{50}$.

L'un des partisans de Kinsey et de la recherche sexologique empirique en Allemagne de l'Ouest, Wolfgang Hochheimer, entrera ouvertement en conflit avec H. Schelsky. Il accuse ce dernier d'être un défenseur des normes morales traditionnelles et de véhiculer des idées qui vont « à l'encontre de la philosophie des Lumières $»^{51}$. W. Hochheimer, à l'inverse de H. Schelsky, admire A. Kinsey, car il montre « la vérité » sur le comportement sexuel humain et dissipe notre « ignorance sur l'étendue réelle des variations du comportement sexuel $»^{52}$. Il n'est pas seul à défendre ce point de vue. Le sociologue von Friedeburg est encore plus engagé dans la défense de la stratégie normaliste flexible. Il rejette les critiques portées par les partisans du protonormalisme. Il reconnaît, certes, que la méthode empirique de recherche ait pu influencer les interviewés, et après publication, le grand public en matière de moralité, mais il considère que ces conséquences sont positives, qu'elles sont « socialement thérapeutiques » et éducatives et qu'en tant que telles, elles peuvent, par exemple, renforcer le concept de mariage ${ }^{53}$. Cependant, pendant les années 1950, un soutien aussi marqué de l'approche normaliste flexible reste isolé.

\section{La « Révolution culturelle » et le normalisme flexible}

Ce n'est qu'à partir du milieu des années 1960 que les sexologues est- et ouestallemand $\cdot e \cdot s$ commencent à pratiquer une recherche plus empirique ${ }^{54}$. En Allemagne

in der DDR. Gesellschaftspolitische Situationen, Sozialisation und Mentalitätsentwicklung in den achtziger Jahren, Opladen, Leske et Budrich, 1991, p. 11 ; voir J. GIESEKE, « Opinion polling behind and across the Iron Curtain: How West and East German pollsters shaped knowledge regimes on communist societies », History of the Human Sciences, 29/4-5, 2016, p. 89-92.

${ }^{49}$ Par exemple dans H. SCHelsky, op. cit., p. 51-52 ; H. Grassel, Jugend, Sexualität, Erziehung, op. cit., p. 51.

${ }^{50}$ A. C. Kinsey, W. B. Pomeroy et C. E. Martin, op. cit., p. 609-616 ; voir Ch. Reinecke, op. cit., p. 35.

${ }_{51}$ W. HochHeImer, « Aufklärung und Gegenaufklärung in der Sexualanthropologie. Zur Problematik der Kontroverse Schelsky - Kinsey », Psyche, 10, 1956/1957, p. 763-784.

52 W. HochHeImer, « Die Kinsey-Berichte », Psyche, 8, 1954/1955, p. 1-38, p. 38.

${ }^{53}$ F. VON Friedeburg, op. cit., p. 15-16.

${ }^{54}$ M. Dannecker, « Die verspätete Empirie », op. cit. ; V. Sigusch, op. cit., p. 430 ; G. GRAU, op. cit., p. 499. 
de l'Ouest, ce phénomène est lié, d'une part, aux changements socio-économiques $\mathrm{du}$ « miracle économique » de l'après-guerre qui valorise les prises de décision rationnelles, scientifiquement fondées, en matière de politique et d'économie et qui demande davantage de données socio-économiques, ce qui conduit à un essor général de la recherche en sciences sociales ${ }^{55}$. D'autre part, ce phénomène est aussi lié au mouvement étudiant de 1968, à sa remise en cause des structures autoritaires et à sa redécouverte des théories sexuelles de Wilhelm Reich ${ }^{56}$. À la conférence de la Société allemande de sexologie, en juin 1969, à Berlin, Reimut Reiche, qui est alors encore doctorant en sociologie, attaque, sur un plan fondamental, l'ancienne génération de sexologues ouest-allemand·e·s sur leur approche normative de la sexologie qui renforce les normes sexuelles traditionnelles et sur leur absence totale de méthodologie en sciences sociales. Reiche veut ouvrir une nouvelle voie pour la recherche en sexologie en Allemagne de l'Ouest qui s'éloigne des recherches cliniques sur les pathologies sexuelles et utilise des méthodes de recherches empiriques et statistiques $^{57}$. Cependant, comme nous l'avons souligné plus haut, en Allemagne de l'Ouest, les sociologues de cette nouvelle génération à laquelle appartiennent Reiche et Amendt, s'ils entreprennent des recherches sexologiques empiriques, se sont d'abord montrés critiques quant aux conséquences de la stratégie normaliste flexible naissante.

En RDA, à la $6^{\mathrm{e}}$ conférence du parti en janvier 1963, une remarque de l'idéologue en chef du Parti socialiste unifié, Kurt Hager, ouvre un nouveau contexte politique pour la recherche en sciences humaines. Il demande, en effet, davantage d'études empiriques sur les problèmes "fondamentaux et généraux » de la société d'Allemagne de l'Est après la construction du mur de Berlin en $1961^{58}$. Cette demande de Hager correspond bien à la vision technocrate de l'État socialiste qu'Ulbricht a présentée à cette même conférence. Ses réformes économiques du « Nouveau système économique » apporteront une plus grande souplesse dans la planification et introduiront une « série de réformes et d'initiatives pour la culture, l'éducation, les femmes et la jeunesse ${ }^{59}$.

${ }^{55}$ Ch. Weischer, op. cit., p. 238-247; W. Süss, “ „Rationale Politik“ durch sozialwissenschaftliche Beratung? Die Projektgruppe Regierungs- und Verwaltungsreform 1966-1975 ", in S. FisCH et W. RudlofF (dir.), Experten und Politik. Wissenschaftliche Politikberatung in geschichtlicher Perspektive, Berlin, Duncker et Humblot, 2004, p. 329-348; A. Schildt, D. Siegfried et K. Ch. Lammers (dir.), Dynamische Zeiten. Die 60er Jahre in den beiden deutschen Gesellschaften, Hambourg, Christians, 2000.

${ }^{56}$ G. AmendT, op. cit., p. 10-22; M. DANNECKer, « Die verspätete Empirie », op. cit., p. 176-177.

${ }^{57}$ R. ReICHE, « Kritik der gegenwärtigen Sexualwissenschaft », op. cit. ; voir V. SigusCH, op. cit., p. 403-407. En 1989, encore, le sexologue Martin Dannecker (« Kann empirische Sexualforschung kritisch sein? », op. cit., p. 211) déplora que, trop fréquemment, les bases empiriques de ce type de recherche soient douteuses et que ces études ne s'appuient pas assez sur la théorie dans leurs interprétations.

${ }^{58}$ Cité d'après G. Grau, op. cit., p. 499. Voir A. Steiner, «Wissenschaft und Politik: Politikberatung in der DDR? », in S. FISCH et W. RudLOFF (dir.), Experten und Politik. Wissenschaftliche Politikberatung in geschichtlicher Perspektive, Berlin, Duncker et Humblot, 2004, p. 101-125.

${ }_{59}$ M. Fulbrook, The People's State: East German Society from Hitler to Honecker, New Haven et Londres, Yale University Press, 2008, p. 38 ; voir A. McDougall, Youth Politics 
C'est dans un tel contexte, et du fait de changements notables en termes de morale sexuelle, qu'un an et demi plus tard, le Parti socialiste unifié publie un Communiqué pour la jeunesse (Jugendkommunique) qui s'engage, dans son titre, à « donner à la jeunesse de la confiance et des responsabilités ${ }^{60}$. Ce Communiqué, le « document le plus radical en matière de politique de la jeunesse de l'histoire du Parti socialiste unifié » (McDougall), reconnaît le fait que les jeunes sont actifs sexuellement avant le mariage et demande à leurs parents et à leurs tuteurs de faire preuve de davantage de compréhension à l'égard de leurs relations de couple, même quand ils ont déjà des relations sexuelles ${ }^{61}$. En 1967, Grassel reconnaît que «pendant longtemps on n'avait pas prêté suffisamment attention à l'importance de la sexualité pour la personne et pour la société » et demande une discussion de fond sur la méthodologie de la recherche sexologique ainsi qu'une évaluation critique des potentiels et des limites de la recherche empirique ${ }^{62}$.

Malgré l'arrêt brutal des réformes entreprises par Ulbricht à peine deux ans plus tard, les sexologues est-allemand·e's se familiarisent avec la recherche en sciences sociales ${ }^{63}$. Au début, leurs enquêtes portent sur les connaissances sexuelles de la jeunesse, mais rapidement, la vision qu'ont les jeunes de la sexualité et leurs comportements sexuels font également l'objet de recherches ${ }^{64}$. Dans les années 1970, les premières enquêtes transversales significatives sur la sexualité sont menées sous

in East Germany: The Free German Youth Movement 1946-1968, Oxford, Clarendon, 2004, p. 153-156 ; Ch. Klessmann, Zwei Staaten, eine Nation. Deutsche Geschichte 1955-1970, Bonn, Bundeszentrale für politische Bildung, $2^{\mathrm{e}}$ éd. révisée, 1997, ch. 9.

${ }^{60}$ « Der Jugend Vertrauen und Verantwortung (1963)», in Dokumente zur Jugendpolitik der DDR, Berlin[-est], Staatsverlag der DDR, 1965, p. 93-94; voir A. McDougall, op. cit., ch. 4 ; U. SchUSTER, « Die SED-Jugendkommuniques von 1961-63. Anmerkungen zur ostdeutschen Jugendpolitik vor und nach dem Mauerbau », Jahrbuch für zeitgeschichtliche Jugendforschung, 1994/1995, p. 58-75 ; Ch. MAHRAD, "Jugendpolitik in der DDR », in W. JAIDE et B. Hille (dir.), Jugend im doppelten Deutschland, Opladen, Westdeutscher Verlag, 1977, p. 208-209.

${ }^{61}$ « Der Jugend Vertrauen und Verantwortung (1963)», op. cit.; A. McDougall, op. cit., p. 154 ; voir J. McLellan, op. cit., p. 25-26 ; M. Fenemore, Sex, Thugs and Rock , n'Roll, op. cit., p. 31-32 et ch. 10 ; D. Herzog, « East Germany's sexual evolution », in K. Pence et P. BetTs (dir.), Socialist Modern: East German Everyday Culture and Politics, Ann Arbor, University of Michigan Press, 2008, p. 71-95 ; D. Herzog, Sex After Fascism, op. cit., p. 195196.

${ }^{62}$ H. Grassel, Jugend, Sexualität, Erziehung, op. cit., p. 39; H. Grassel, «Zur Problematik der Methoden der Sexualforschung ", op. cit., p. 381.

${ }_{63}$ J. AdAm, E. FikentSCher et K.-H. Liebner, « Untersuchungen zur sexuellen Entwicklung (Fragebogenerhebung an Medizinstudenten der DDR durch eine Arbeitsgruppe unter Leitung von Helmut Rennert): 1. Mitteilung: Planung und Durchführung der Befragung », Wissenschaftliche Zeitschrift der Martin-Luther-Universität Halle-Wittenberg: Mathematischnaturwissenschaftliche Reihe, 25/3, 1976, p. 81-91.

${ }^{64}$ H. Grassel, Jugend, Sexualität, Erziehung, op. cit.; H. BrüCKnER, Das Sexualwissen unserer Jugend, dargestellt als Beitrag zur Erziehungsplanung, Berlin[-est], VEB Deutscher Verlag der Wissenschaften, 1968 ; H. RENNERT, «Untersuchungen über die sexuelle Entwicklung der Jugend in der DDR », Wissenschaftliche Zeitschrift der Universität Rostock: MathematischNaturwissenschaftliche Reihe, 17, 1968, p. 701-708. 
les auspices de l'Institut central des études sur la jeunesse, à Leipzig (Zentralinstitut für Jugendforschung, fondé en 1966) ${ }^{65}$. Cependant, ni le gouvernement de la RDA ni le Parti socialiste unifié ne reconnaissent officiellement l'implication de l'Institut dans les recherches empiriques en matière de sexualité, même si l'Institut présente bien ses rapports au gouvernement central, via le Bureau des affaires de la jeunesse ${ }^{66}$.

Il demeure difficile de mener des recherches sexologiques empiriques en RDA et l'opposition à la diffusion de leurs résultats auprès du grand public perdure ${ }^{67}$. L'Institut est cependant toujours en mesure de publier les résultats des sondages sur la sexualité, bien que l'aspect empirique de ce type de recherche demeure généralement secret $^{68}$. Dans les années 1980, le psychologue et directeur de l'Institut, le professeur Walter Friedrich, finit par reconnaître les apports de Kinsey et de son équipe. Il admet ainsi que Kinsey a apporté « des preuves, bien documentées d'un point de vue empirique, sur des relations, des différences et des similarités entre différents groupes sociaux » et leurs comportements sexuels ${ }^{69}$. Bien qu'il reproche à Kinsey « sa tendance à biologiser le comportement sexuel humain » et à ne pas parvenir à rendre compte de la dimension psychologique de la sexualité, Friedrich reconnaît que les rapports Kinsey constituent les enquêtes les plus importantes du $\mathrm{XX}^{\mathrm{e}}$ siècle sur le comportement sexuel ${ }^{70}$.

\section{Résultats des enquêtes sur la sexualité et normalisation de la sexualité de la jeunesse pendant la guerre froide}

Une synthèse des principaux résultats des enquêtes sur la sexualité de la jeunesse permet d'illustrer à quel point le comportement sexuel des jeunes devient «normal » après la Seconde Guerre mondiale. De même, une telle synthèse montre bien l'étendue des changements qui se produisent alors en matière de normes morales dans les deux Allemagnes. La toute première enquête sur la sexualité menée par l'institut de sondage Allensbach pour Wochenend en 1949 faisait déjà apparaître un décalage entre les conceptions des jeunes sur la morale et les comportements sexuels, d'une part, et les normes sexuelles officielles de la société ouest-allemande, d'autre part. Par exemple, l'enquête a révélé que les relations sexuelles préconjugales étaient devenues relativement fréquentes. Seules $22 \%$ des femmes considéraient leur virginité comme essentielle au moment du mariage mais près d'un tiers d'entre elles tenaient un

${ }^{65}$ K. STARKe, Junge Partner. Tatsachen über Liebesbeziehungen im Jugendalter, Leipzig, Jena et Berlin, Urania, 1980 ; K. StARKe et W. Friedrich (dir.), Liebe und Sexualität bis 30, Berlin[-est], VEB Deutscher Verlag der Wissenschaften, 1984.

${ }^{66}$ K. Starke et K. Weller, «Partner- und Sexualforschung », in W. Friedrich, P. Förster et K. StARKe (dir.), Das Zentralinstitut für Jugendforschung in Leipzig 1966-1990. Geschichte, Methoden, Erkenntnisse, Berlin, Ost, 1999, p. 397.

${ }^{67}$ S. Schnabl, Intimverhalten, Sexualstörungen, Persönlichkeit, Berlin[-est], Deutscher Verlag der Wissenschaften, 1973, p. 49-50.

${ }_{68}$ W. Friedrich, op. cit., p. 14, 23 ; K. Starke et K. Weller, op. cit., p. 397 ; voir M. Fenemore, Sex, Thugs and Rock 'n' Roll, op. cit., p. 32.

${ }^{69}$ W. Friedrich, « Anmerkungen zur Geschichte der Sexualforschung », in K. STARKe et W. Friedrich (dir.), Liebe und Sexualität bis 30, Berlin[-est], VEB Deutscher Verlag der Wissenschaften, 1984, p. 38.

${ }^{70}$ Ibid., p. 35-36, citation p. 38-39. 
manque d'expérience sexuelle avant le mariage pour préjudiciable. Plus de la moitié des interviewés estimaient qu'il était nécessaire d'avoir eu des expériences sexuelles avant de se marier, ce que confirmaient les données sur leur comportement sexuel où près de $90 \%$ des hommes et $70 \%$ des femmes reconnaissaient avoir connu des " relations intimes » préconjugales, et ce, principalement avec quelqu'un d'autre que leur futur mari ou leur future femme. Seuls les répondant·e·s âgé·e·s de 50 ans et plus considéraient comme répréhensibles les relations préconjugales. Bien que les répondant $\cdot \mathrm{e} \cdot \mathrm{s}$, dans leur vaste majorité, disaient croire dans l'amour et dans le mariage, $30 \%$ des répondants et $14 \%$ des répondantes reconnaissaient avoir eu leur première expérience sexuelle avant 17 ans et, respectivement, $38 \%$ et $24 \%$ avant 18 ans $^{71}$. Le magazine Wochenend conclut donc, en 1949, que « les contemporains sont dans leur vaste majorité ni prudes ni romantiques ${ }^{72}$. Ni ces positions ni ces comportements ne correspondaient pourtant aux normes de la moralité officielle de l'époque. La conception de l'homosexualité demeurait négative avec $39 \%$ des répondant·e's la percevant comme une maladie et $48 \%$ d'entre eux $\cdot e l l e s$ comme un vice - un sujet que Wochenend n'ose d'ailleurs pas aborder ${ }^{73}$.

Comme le montre la première enquête représentative sur la sexualité des adolescents, menée par Volkmar Sigush et Gunter Schmidt, l'amour et la fidélité continuent d'être des valeurs primordiales pour les 302 élèves garçons et les 300 élèves filles âgés de 16 à 17 ans qu'ils ont interrogés dans les villes d'Allemagne de l'Ouest entre mai et juin 1970 . Cependant, les adolescent $\cdot e \cdot s$ deviennent actif $\cdot v e \cdot s$ sexuellement beaucoup plus tôt que dans les générations précédentes. Un tiers des élèves affirment avoir eu des relations sexuelles pendant les 12 mois précédents, un autre tiers reconnaissent pratiquer des caresses érotiques et le tiers restant disent se masturber ou avoir été sexuellement abstinent $\cdot e \cdot s^{74}$. Le comportement sexuel des adolescent·e's d'Allemagne de l'Ouest change rapidement pendant les années 1960. Alors que seuls 10 à $20 \%$ des étudiant·e·s né·e·s pendant les années 1930 ou 1940 avaient connu leur première expérience sexuelle avant 18 ans, ce pourcentage monte à $47 \%$ des étudiants et à $61 \%$ des étudiantes durant le début des années $1950^{75}$. Pendant les décennies suivantes, ces chiffres n'augmentent que légèrement. Plus notables sont les changements du comportement sexuel des adolescentes : pendant les années 1970,

${ }^{71}$ « Urmacht Liebe in unserer Zeit: Liebe vor der Ehe? », Wochenend, $\mathrm{n}^{\circ} 2,12$ janvier 1950, p. 6 ; H. W., « Eine Frage mit Hintergrund: Soll die Frau unberührt in die Ehe gehen? », Wochenend, n 3, 19 janvier 1950, p. 6 ; H. W., « Das bleibende Ziel: der Mensch. Die Ergebnisse der großen Sexualanalyse », Wochenend, $\mathrm{n}^{\circ}$ 7, 16 février 1950, p. 6 ; «Intimsphäre », op. cit., p. 592 ; F. vON FRIEDEBURG, op. cit., p. 19-22, 24-25, 56-57, 86, 88-89.

${ }^{72}$ H. W., «Urmacht Liebe in unserer Zeit », op. cit.

73 « Intimsphäre », op. cit., p. 591 ; F. vON FrIEDEBurG, op. cit., p. 87.

74 V. Sigusch et G. Schmidt, Jugendsexualität. Dokumentation einer Untersuchung, Stuttgart, Enke, 1973, p. 8-9, 11-16, 71-76, 153, 185.

75 G. Schmidt, «Zur Sozialgeschichte jugendlichen Sexualverhaltens in der zweiten Hälfte des 20. Jahrhunderts », in C. Bruns et T. WALter (dir.), Von Lust und Schmerz. Eine historische Anthropologie der Sexualität, Cologne, Weimar et Vienne, Böhlau, 2004, p. 314315,325 . 
elles deviennent sexuellement actives à un âge moins avancé que les adolescents. Jusqu'à la fin des années 1960, c'est l'inverse qui se produisait ${ }^{76}$.

Si l'on excepte quelques enquêtes plus spécialisées, isolées et de moindre ampleur, on peut considérer que la première enquête globale en Allemagne de l'Est sur les conceptions de la sexualité chez les jeunes et sur leur comportement sexuel a lieu en 1972-1973 ${ }^{77}$. Pour son enquête, le sexologue Kurt Starke, à l'Institut central d'études sur la jeunesse (Zentralinstitut für Jugendforschung, ZIJ), s'appuie sur des questionnaires que plus de 2700 répondant $\cdot e \cdot s$ (étudiant $\cdot e \cdot s$, apprenti·e $\cdot s$, jeunes travailleur·euse $\cdot s$ ) remplissent de façon anonyme. L'Institut conduit une deuxième grande étude similaire en 1979-1980 où près de 5500 citoyen'ne's de RDA âgé $\cdot e \cdot s$ de 16 à 30 ans sont invité $\cdot e \cdot s$ à répondre à un questionnaire écrit ${ }^{78}$.

Comparant les résultats de ces deux enquêtes, le sexologue de RDA K. Starke note « des changements significatifs dans le rapport de la jeunesse à l'amour et à la sexualité » qui lui semblent correspondre à la tendance générale à la « libéralisation » dans les pays industrialisés ${ }^{79}$. Mais Starke identifie également, dans les positions sur la sexualité et les comportements sexuels des jeunes Allemand·e·s de l'Est, une spécificité qui les différencie de leurs congénères de l'Ouest. Cette spécificité réside, notamment, dans « l'étrange connexion entre amour et sexualité qu'établit, intellectuellement et affectivement, la jeunesse de RDA », le fait qu'ils soient plus tournés vers la famille et le couple, leur âge moins élevé lors du premier rapport, leur moindre fréquence de masturbation et leur " acceptation complète des relations sexuelles préconjugales $»^{80}$. En effet, $98 \%$ des répondants et $97 \%$ des répondantes n'ont aucune objection à l'activité sexuelle préconjugale ${ }^{81}$. À l'âge de 16 ans, près de $60 \%$ des répondant·e·s affirment avoir eu déjà des expériences sexuelles, à l'exception de rapports sexuels en tant que tels ${ }^{82}$. En comparant les résultats d'un certain nombre de petites enquêtes des années 1950 et 1960, Hans-Joachim Ahrendt estime qu'entre les années 1950 et les années 1980, l'âge moyen du premier rapport sexuel a chuté de 4 à 5 ans, d'un peu plus de 20 ans au milieu des années 1950 à 18 ans au milieu des années 1970 et à 16-17 ans une décennie plus tard ${ }^{83}$. Il considère que les changements dans la législation sur l'avortement et un accès plus facile à la contraception ont grandement contribué à cette baisse ${ }^{84}$. Les études de grande ampleur de l'Institut central des études sur la jeunesse montrent que si en 1973, $12 \%$ des adolescent·e·s de 16 ans ont eu des

${ }^{76}$ Ibid., p. 315.

77 K. Starke, op. cit. ; voir J. Adam, E. Fikentscher et K. Liebner, op. cit., p. 81.

${ }^{78}$ K. Starke et W. Friedrich (dir.), Liebe und Sexualität bis 30, Berlin[-est], VEB Deutscher Verlag der Wissenschaften, 1984.

${ }^{79}$ K. Starke, « Jugendsexualität », in J. S. Hohmann (dir.), Sexuologie in der DDR, Berlin, Dietz, 1991, p. 210.

${ }^{80}$ Ibid.

${ }^{81}$ K. STARKe, Junge Partner, op. cit., p. 75.

${ }^{82}$ Ibid., p. 116.

${ }^{83}$ H.-J. Ahrendt, Geschlechtliche Entwicklung, Sexualverhalten und Kontrazeption 15bis 17jähriger weiblicher Jugendlicher (thèse d'habilitation non publiée), Magdeburg, 1985, p. $50-51$.

${ }^{84}$ Ibid., p. 52-53. 
relations sexuelles, ce pourcentage a presque doublé pour atteindre $22 \%$ en $1977^{85}$. En moins d'une décennie, l'âge moyen du premier rapport sexuel tombe de 17,5 ans chez les jeunes travailleur'euse's et 18,3 ans chez les étudiant'e's en 1973, à 16,9 ans, chez tous les répondant·e·s, hommes comme femmes, en $1977^{86}$. Aussi, selon Starke, la sexualité des jeunes devient généralement acceptée pendant les années 1970 et les citoyen·ne·s de RDA commencent à décider de leur vie sexuelle sans que l'État ne s'immisce dans la sphère privée que constitue la chambre à coucher des couples ${ }^{87}$.

Les résultats de ces vastes enquêtes sur la sexualité de la jeunesse paraissent non seulement dans des publications universitaires en Allemagne de 1'Est, mais un plus large public peut aussi en prendre connaissance, bien que dans une moindre mesure qu'en Allemagne de l'Ouest. La publication de la première étude de Starke, Jeunes Partenaires, connaît ainsi deux éditions avec un tirage de 90000 exemplaires et sa seconde étude devient un succès de librairie avec quatre éditions et 250000 exemplaires vendus $^{88}$. Si l'on en juge d'après le style de l'écriture, les illustrations et la façon dont Starke s'adresse directement au lectorat, Jeunes Partenaires est clairement destiné au grand public. Dans sa préface, Friedrich, le directeur de l'Institut central des études sur la jeunesse, confirme que Jeunes Partenaires n'est pas seulement écrit à l'intention des parents, des professeur·e's, des éducateur·rice·s, etc., mais aussi des jeunes eux-mêmes ${ }^{89}$.

En Allemagne de l'Ouest, les résultats des enquêtes sur la sexualité publiés initialement dans un contexte universitaire atteignent également le grand public dans la mesure où les médias s'empressent de les relayer. Ainsi, le magazine Eltern («Parents ») prépublie les résultats de l'enquête de Sigusch et Schmidt de 1970, avec un intérêt marqué pour le comportement des jeunes en matière de contraception ${ }^{90}$. Si les magazines est-allemands destinés à la jeunesse (comme Neues Leben « Nouvelle Vie ») ne commandent pas leurs propres enquêtes sur la sexualité pas plus qu'ils ne commentent de façon significative les résultats des enquêtes ${ }^{91}$, la presse jeunesse d'Allemagne de l'Ouest, comme Bravo, le magazine le plus populaire, fait constamment référence aux données statistiques sur les comportements sexuels des jeunes. Par exemple, en 1968, Bravo rapporte, dans une série de huit articles sur « la

${ }^{85}$ K. STARKe, Junge Partner, op. cit., p. 120.

${ }^{86}$ K. Starke, « Der erste Geschlechtsverkehr », in K. Starke et W. Friedrich (dir.), Liebe und Sexualität bis 30, Berlin[-est], VEB Deutscher Verlag der Wissenschaften, 1984, p. 137.

${ }^{87}$ K. STARKe, «Die ungewöhnliche gewöhnliche Sexualität in der DDR », in L. Elm, D. Keller et R. MOceK (dir.), Ansichten zur Geschichte der DDR, vol. 8, 2 partie, Eggersdorf, Kirchner, 1997, p. 163.

${ }^{88}$ K. Starke, Junge Partner, op. cit. ; K. Starke et W. Friedrich, Liebe und Sexualität bis 30, op. cit.

${ }^{89}$ W. Friedrich, "Zum Geleit », in K. Starke, Junge Partner. Tatsachen über Liebesbeziehungen im Jugendalter, Leipzig, Jena et Berlin, Urania, 1980, p. 9.

90 «Sexualreport [2 ${ }^{\mathrm{e}}$ partie] », Eltern, 1970, no 10, p. 34-43, p. 38 ; P. BocCARIUS, «Sexualreport [2 $2^{\mathrm{e}}$ partie] $»$, Eltern, 1970, nº 11, p. 50-60.

${ }^{91}$ En 1966, le magazine de santé Deine Gesundheit (Ta Santé) essaya de sonder 50 de ses abonné $\cdot \mathrm{e} \cdot \mathrm{s}$ sur leur première expérience sexuelle et leurs relations amoureuses, mais seul $\cdot \mathrm{e} \cdot \mathrm{s}$ la moitié d'entre eux elles répondirent (dont 5 personnes refusèrent de répondre aux questions). G. MisGelD, « Einer für viele », Deine Gesundheit, 12/6, 1966, p. 182-185. 
sexualité dans les écoles », que $60 \%$ des garçons âgés de 15 à 18 ans ont une petite amie, que $55 \%$ des filles âgées de 14 à 18 ans ont un petit ami et qu'en outre, $20 \%$ des filles ont déjà eu des rapports sexuels ${ }^{92}$. Bravo commande également ses propres enquêtes sur la sexualité et les relations amoureuses. Dans une plus large mesure que leurs homologues est-allemands, les adolescent·e's ouest-allemand·e's sont régulièrement invité·e·s à comparer leur conception de la sexualité, leurs expériences et comportements sexuels avec ceux de leurs pairs.

Certes, la recherche d'Allemagne de l'Est a évolué vers une sorte de normalisme flexible pendant les années 1970 et les limites, auparavant immuables, de ce qui était acceptable en matière de moralité et de comportement sexuel sont devenues nettement moins nettes qu'elles ne l'avaient été pendant les années 1950. On constate pourtant des différences entre les deux Allemagnes en ce qui concerne la communication au grand public, et à la jeunesse en particulier, des données obtenues par les enquêtes sur la sexualité. Cependant, malgré ces différences, la sexualité adolescente semble devenue acceptable et «normale » pendant les années 1970, des deux côtés du rideau de fer.

\section{Conclusion}

Comme nous l'avons vu, les sexologues d'Allemagne de l'Est et de l'Ouest se tournent vers la recherche sexologique empirique et une stratégie normaliste flexible à la fin de la Seconde Guerre mondiale, avec du retard sur leurs collègues du monde anglo-américain. Jusqu'à tard dans les années 1960, la recherche sexologique empirique reste controversée du point de vue méthodologique, dans les deux pays. Les principaux sexologues, spécialistes des sciences sociales et autres commentateurs s'inquiètent surtout de l'effet normalisateur de ces données statistiques sur la morale et les comportements sexuels. Ils craignent que cet effet normalisateur affaiblisse les normes de la morale sexuelle et les rôles de genre de l'époque et donc qu'il diminue, voire qu'il détruise tout à fait les limites rigides de l'acceptabilité que la stratégie protonormaliste avait maintenues. Cependant, à partir des années 1970, les deux pays deviennent des " sociétés qui reposent sur l'analyse de données » modernes et leurs gouvernements dépendent de plus en plus des données statistiques pour mener leur action politique. Pour Jürgen Link, l'introduction de la recherche sexologique empirique en Allemagne de l'Est et de l'Ouest et l'acceptation qu'elle provoqua d'une stratégie normaliste flexible représente une "révolution culturelle " qui permit au normalisme flexible de s'imposer ${ }^{93}$.

Les enquêtes sur la sexualité contribuent donc de façon significative à la normalisation de la sexualité adolescente, probablement davantage en Allemagne de l'Ouest où les magazines pour adolescent $\cdot e \cdot s$ vulgarisent, à un niveau sans précédent, les données statistiques sur les conceptions morales de la sexualité et sur les pratiques sexuelles. La normalisation de la sexualité adolescente a des conséquences considérables des deux côtés du rideau de fer puisqu'elle va de pair avec un assouplissement du contrôle social et du code traditionnel de morale sexuelle des

\footnotetext{
92 «Sex in der Schule », Bravo, 1968, n 33, p. 16.

93 J. Link, Versuch über den Normalismus, op. cit., p. 23.
} 
années 1950. Le discours de la morale et des valeurs familiales chrétiennes, à l'Ouest, et la morale socialiste, à l'Est - qui mettait l'accent sur la dimension politique du mariage, de la famille et de la responsabilité des citoyen·ne·s vis-à-vis de l'État -, perdent de plus en plus de terrain chez les jeunes pendant les années 1960 et 1970, et là encore, davantage à l'Ouest qu'à l'Est. Toutefois, on peut identifier, avec Mark Fenemore, « un bouleversement sismique en ce qui concerne la sexualité » ou, avec Dagmar Herzog, une " évolution sexuelle » à partir de la moitié des années 1960 en $\mathrm{RDA}^{94}$. Les enquêtes sur la sexualité font apparaître à quel point les conceptions de la sexualité et les comportements sexuels des Allemand·e·s de l'Est se sont éloignés de la morale socialiste officielle, sans toutefois mettre en péril les " institutions fondamentales de la société socialiste que constituaient le mariage et la famille ${ }^{95}$.

Depuis la fin des années 1960, les limites protonormalistes de la sexualité, auparavant strictes et immuables, sont devenues bien plus flexibles, des deux côtés du rideau de fer, malgré les différences fondamentales entre les régimes politiques et l'ordre social qui y règne. Il n'est plus « normal», par exemple, de ne pas se masturber ou de ne pas avoir de relations sexuelles avant le mariage. Il n'est plus « normal» d'interdire aux jeunes l'accès à la contraception. Au même moment, les jeunes d'Allemagne de l'Est et de l'Ouest - souvent encore mineurs - se montrent de plus en plus responsables en ce qui concerne leur comportement sexuel et leurs pratiques, par exemple, dans leur utilisation de la contraception ou en matière de compétences érotiques ${ }^{96}$. Avec la « révolution culturelle» du normalisme flexible, un nouveau code de moralité sexuelle voit le jour. Ce code est fondé sur la capacité de partenaires, égaux et responsables, de négocier leurs activités sexuelles ${ }^{97}$. Dans cette " éthique de la négociation », la seule contrainte en matière d'activités sexuelles réside dans l'accord auquel le couple doit parvenir sur les questions concernant sa vie sexuelle. Cette nouvelle éthique de négociation permet d'atteindre le niveau de flexibilité exigé par la culture du normalisme flexible, à l'Ouest comme à l'Est. Cette flexibilité existe donc bel et bien dans les deux Allemagnes avec, seulement, des différences de degrés, mais non de principe, puisque l'Allemagne de l'Est continue d'exiger de sa jeunesse qu'elle se conforme de façon significative aux valeurs de l'État socialiste.

${ }^{94}$ M. Fenemore, Sex, Thugs and Rock ,n'Roll, op. cit., p. 33 ; D. Herzog, « East Germany's sexual evolution », in K. Pence et P. Betts, Socialist Modern: East German Everyday Culture and Politics, Ann Arbor, University of Michigan Press, 2008 ; voir K. STARKe, « Die ungewöhnliche gewöhnliche Sexualität in der DDR », op. cit., p. 169-171.

${ }_{95}$ M. Fenemore, Sex, Thugs and Rock ' $n$ 'Roll, op. cit., p. 33.

${ }^{96}$ L. SAUERTEIG, «From the fear of conception to the management of sex: birth control in West German sex education material, c. 1945-1980 », in L. NiETHAmmER et S. SATJukow (dir.), „Wenn die Chemie stimmt “...: Geschlechterbeziehungen und Geburtenkontrolle im Zeitalter der „Pille “/ Gender Relations and Birth Control in the Age of the "Pill", Göttingen, Wallstein, 2016, p. 211-241 ; L. SAUERTEIG, « Die Herstellung des sexuellen und erotischen Körpers », op. cit.

${ }^{97}$ L. Sauerteig, "From the fear of conception to the management of sex », op. cit., p. $237-241$. 


\section{Bibliographie}

Adam, J., Fikentscher, E. et Liebner, K.-H., « Untersuchungen zur sexuellen Entwicklung (Fragebogenerhebung an Medizinstudenten der DDR durch eine Arbeitsgruppe unter Leitung von Helmut Rennert): 1. Mitteilung: Planung und Durchführung der Befragung », Wissenschaftliche Zeitschrift der Martin-LutherUniversität Halle-Wittenberg: Mathematisch-naturwissenschaftliche Reihe, 25/3, 1976, p. 81-91.

Ahrendt, H.-J., Geschlechtliche Entwicklung, Sexualverhalten und Kontrazeption 15- bis 17jähriger weiblicher Jugendlicher (thèse d'habilitation non publiée), Magdeburg, 1985.

Amendt, G., " Empirie, Emanzipation und Sexualforschung », in Schmidt, G., Sigusch, V. et Becker, S., « Bemerkungen zur Debatte über Bürger-Prinz », Z. Sexualforsch., 4, 1991, p. 265-270.

Beiträge zur Geschlechtserziehung, partie I : Biologie der Fortpflanzung, Cologne, 1967.

BRÜCKNER, H., Das Sexualwissen unserer Jugend, dargestellt als Beitrag zur Erziehungsplanung, Berlin[-est], VEB Deutscher Verlag der Wissenschaften, 1968.

BüHRMANN, A. B., « Die gesellschaftlichen Konsequenzen der Wissensproduktion. Zum Verhältnis von (Sexual-)Wissenschaften und gesellschaftlichen Normalisierungsmechanismen ", in Ferdinand Pretzel, U. et SeeK, A. (dir.), Verqueere Wissenschaft? Zum Verhältnis von Sexualwissenschaft und Sexualreformbewegung in Geschichte und Gegenwart, Münster, Lit, 1998, p. 213-228.

Converse, J. M., Survey Research in the United States: Roots and Emergence 18901960, Berkeley et Londres, University of California Press, 1987.

DANneCKer, M., « Die verspätete Empirie. Anmerkungen zu den Anfängen der Deutschen Gesellschaft für Sexualforschung », Z. Sexualforsch., 14, 2001, p. 166-180.

FEnEmore, M., « The growing pains of sex education in the German Democratic Republic (GDR), 1945-1969 », in SAuerteig, L. et Davidson, R. (dir.), Shaping Sexual Knowledge: A Cultural History of Sex Education in Twentieth Century Europe, Londres et New York, Routledge, 2009, p. 71-90.

Fenemore, M., Sex, Thugs and Rock ' $n$ ' Roll: Teenage Rebels in Cold-War East Germany, New York et Oxford, Berghahn Books, 2009.

Fredericia, W. [Walter Petwaidic], « Kinsey über die amerikanische Frau: Die Vivisektion des Menschen mit dem Messer des modernen Zoologen », Die Zeit, 19 novembre $1953, \mathrm{n}^{\circ} 47$.

FRIEDRICH, W., « DDR-Jugendforschung - zwischen wissenschaftlichem Anspruch und politischer Bevormundung », in FrIEDRICH, W. et GRIESE, H. (dir.), Jugend und Jugendforschung in der DDR. Gesellschaftspolitische Situationen, Sozialisation und Mentalitätsentwicklung in den achtziger Jahren, Opladen, Leske et Budrich, 1991, p. 11-26.

Fulbrook, M., The People's State: East German Society from Hitler to Honecker, New Haven et Londres, Yale University Press, 2008. 
Gerhard, U., Link, J. et Schulte-Holtey, E. (dir.), Infografiken, Medien, Normalisierung. Zur Kartografie politisch-sozialer Landschaften, Heidelberg, Synchron, 2001.

Giese, H. et Schmidt, G., Studentensexualität. Verhalten und Einstellung. Eine Umfrage an 12 westdeutschen Universitäten, Reinbek, Rowohlt, 1968.

GIESEKE, J., « Opinion polling behind and across the Iron Curtain: How West and East German pollsters shaped knowledge regimes on communist societies », History of the Human Sciences, 29/4-5, 2016, p. 77-98.

Grassel, H., «Zur Problematik der Methoden der Sexualforschung», Wissenschaftliche Zeitschrift der Universität Rostock: Gesellschafts- und sprachwissenschaftliche Reihe, 16, 1967, p. 381-394.

Grassel, H., Jugend, Sexualität, Erziehung. Zur psychologischen Problematik der Geschlechtserziehung, Berlin[-est], Staatsverlag der DDR, 1967.

Grau, G., "Sexualwissenschaft in der DDR: Ein Resümee », in Sigusch, V., Geschichte der Sexualwissenschaft, Francfort et New York, Campus, 2008, p. 487-509.

Grossmann, A., Reforming Sex: The German Movement for Birth Control and Abortion reform, 1920-1950, New York et Oxford, Oxford University Press, 1995.

Heineman, E. D., Before Porn was Legal: The Erotica Empire of Beate Uhse, Chicago, Ill., University of Chicago Press, 2011.

Herzog, D., « The reception of the Kinsey reports in Europe », Sexuality and Culture, 10/1, 2006, p. 39-48.

Herzog, D., « East Germany's sexual evolution », in Pence, K. et Betts, P. (dir.), Socialist Modern: East German Everyday Culture and Politics, Ann Arbor, University of Michigan Press, 2008, p. 71-95.

Herzog, D., Sex After Fascism: Memory and Morality in Twentieth-Century Germany, Princeton et Oxford, Princeton University Press, 2005.

Hesse, P. G., « Die Anfänge der Sexuologie in der DDR », in Hohmann, J. S. (dir.), Sexuologie in der DDR, Berlin, Dietz, 1991, p. 51-61.

HochHeIMeR, W., « Aufklärung und Gegenaufklärung in der Sexualanthropologie. Zur Problematik der Kontroverse Schelsky - Kinsey », Psyche, 10, 1956/1957, p. 763-784.

Hochheimer, W., « Die Kinsey-Berichte », Psyche, 8, 1954/1955, p. 1-38.

Hoffmann, H.-J. et Klemm, P. G., Ein offenes Wort. Ein Buch über die Liebe, Berlin [-est], Neues Leben, 1956.

Igo, S. E., The Averaged American: Surveys, Citizens, and the Making of a Mass Public, Cambridge, Mass., et Londres, Harvard University Press, 2007.

KInsey, A. C. et al., Das sexuelle Verhalten der Frau, [Sexual Behavior in the Human Female, Philadelphie et Londres, Saunders, 1953], [trad. de M. Baacke et al.], Berlin et Francfort, Fischer, 1954, réimpr. 1967.

Kinsey, A. C., Pomeroy, W. B. et Martin, C. E., Das sexuelle Verhalten des Mannes, [Sexual Behaviour in the Human Male, Philadelphie, Saunders, 1948], [trad. de M. von Eckardt-Jaffé, M. Baacke et W. Seemann], Berlin et Francfort, Fischer, 1955, réimpression 1967. 
Klessmann, Ch., Zwei Staaten, eine Nation. Deutsche Geschichte 1955-1970, Bonn, Bundeszentrale für politische Bildung, 2e éd. révisée, 1997.

LiebeKneCht, M., « „Wir ertrinken in der Sex-Welle“. Hans Giese und der öffentliche Sexualitätsdiskurs in den sechziger Jahren », Zeitgeschichte in Hamburg, 2016, p. 52-67.

LiebeKneCHT, M., « Sexualität als Gegenstand der Zeitgeschichtsforschung und der Sexualwissenschaft in der frühen Bundesrepublik », Zeitschrift für Sexualforschung [Z. Sexualforsch.], 28, 2015, p. 132-148.

LINK, J., "From the "power of the norm" to "flexible normalism": considerations after Foucault » [trad. de M. M. Hall], Cultural Critique, 2004, nº 57, p. 14-32.

LINK, J., " On the contribution of normalism to modernity and postmodernity » [trad. de M. M. Hall], Cultural Critique, 2004, nº 57, p. 33-46.

LINK, J., « The normalistic subject and its curves: on the symbolic visualization of orienteering data » [trad. de M. M. Hall], Cultural Critique, 2004, nº 57, p. 47-67.

LinK, J., Versuch über den Normalismus. Wie Normalität produziert wird, Göttingen, Vandenhoeck et Ruprecht, 1997, 4éd., 2009.

DANNECKER, M., « Kann empirische Sexualforschung kritisch sein? Zum Andenken an Alfred C. Kinsey », Z. Sexualforsch., 2, 1989, p. 207-215.

Mahrad, Ch., " Jugendpolitik in der DDR », in JAIDE, W. et Hille, B. (dir.), Jugend im doppelten Deutschland, Opladen, Westdeutscher Verlag, 1977, p. 195-225.

McDougall, A., Youth Politics in East Germany: The Free German Youth Movement 1946-1968, Oxford, Clarendon, 2004.

McLellan, J., Love in the Time of Communism: Intimacy and Sexuality in the GDR, Cambridge et New York, Cambridge University Press, 2011.

Misgeld, G., « Einer für viele », Deine Gesundheit, 12/6, 1966, p. 182-185.

Neubert, R., Das neue Ehebuch. Die Ehe als Aufgabe der Gegenwart und Zukunft, Rudolstadt, Greifenverlag, 1957, 20éd. révisée, 1972.

Neubert, R., Die Geschlechterfrage. Ein Buch für junge Menschen, Rudolstadt, Greifenverlag, $4^{\mathrm{e}}$ éd. révisée, 1956.

NiKolow, S., « Imaginäre Gemeinschaften. Statistische Bilder der Bevölkerung », in Hessler, M. (dir.), Konstruierte Sichtbarkeiten. Wissenschafts- und Technikbilder seit der Frühen Neuzeit, Munich, Fink, 2006, p. 263-278.

Poiger, U. G., Jazz, Rock, and Rebels: Cold War Politics and American Culture in a Divided Germany, Berkeley, Los Angeles et Londres, University of California Press, 2000.

Protokoll der Verhandlungen des V. Parteitages der Sozialistischen Einheitspartei Deutschlands: 10. bis 16. Juli 1958 in der Werner-Seelbinder-Halle zu Berlin, 2 vol., Berlin[-est], Dietz, 1959.

ReICHE, R., "Kritik der gegenwärtigen Sexualwissenschaft », in ScHMIDT, G., Sigusch, V. et Schorsch, E. (dir.), Tendenzen der Sexualforschung, Stuttgart, Enke, 1970, p. 1-9.

Reiche, R., « Die Aufnahme der Kinsey-Berichte », Vorgänge, 5, 1966, p. 364-372.

REINECKE, Ch., « Statistiken der Liebe oder: Dr. Kinsey fragt die Frauen. Umfrageforschung und ihre mediale Vermarktung in transnationaler Perspektive », Comparativ, 21/4, 2011, p. 29-44. 
RENNERT, H., «Untersuchungen über die sexuelle Entwicklung der Jugend in der DDR », Wissenschaftliche Zeitschrift der Universität Rostock: MathematischNaturwissenschaftliche Reihe, 17, 1968, p. 701-708.

Sauerteig, L., " From the fear of conception to the management of sex: birth control in West German sex education material, c. 1945-1980 », in Niethammer, L. et Satuukow, S. (dir.), ,, Wenn die Chemie stimmt “...: Geschlechterbeziehungen und Geburtenkontrolle im Zeitalter der ,Pille“ / Gender Relations and Birth Control in the Age of the "Pill", Göttingen, Wallstein, 2016, p. 211-241.

Sauerteig, L., « Junge oder Mädchen - Frau oder Mann? Der heterosexuelle Körper und die Herstellung visueller Selbstverständlichkeiten in der Sexualaufklärung im 20. Jahrhundert », WerkstattGeschichte, 16/47, 2007, p. 40-60.

Sauerteig, L., « Die Herstellung des sexuellen und erotischen Körpers in der westdeutschen Jugendzeitschrift BRAVO in den 1960er und 1970er Jahren », Medizinhistorisches Journal, 42, 2007, p. 142-179.

SAuerteig, L., "Representations of pregnancy and childbirth in (West) German sex education books, 1900s-1970s », in Sauerteig, L., Davidson, R. ; et (dir.), Shaping Sexual Knowledge: A Cultural History of Sex Education in Twentieth Century Europe, Londres et New York, Routledge, 2009, p. 120-160.

Schelsky, H., Soziologie der Sexualität. Über die Beziehungen zwischen Geschlecht, Moral und Gesellschaft, Hambourg, Rowohlt TB, 1955.

Schildt, A., Siegfried, D. et Lammers, K. Ch. (dir.), Dynamische Zeiten. Die 60er Jahre in den beiden deutschen Gesellschaften, Hambourg, Christians, 2000.

SCHMIDT, G., " Kinsey's unspoken truth: on how the Kinsey reports were received in West Germany », Sexualities, 1, 1998, p. 100-103.

Schmidt, G., « Zur Sozialgeschichte jugendlichen Sexualverhaltens in der zweiten Hälfte des 20. Jahrhunderts », in Bruns, C. et Walter, T. (dir.), Von Lust und Schmerz. Eine historische Anthropologie der Sexualität, Cologne, Weimar et Vienne, Böhlau, 2004, p. 312-325.

Schnabl, S., Intimverhalten, Sexualstörungen, Persönlichkeit, Berlin[-est], Deutscher Verlag der Wissenschaften, 1973, p. 49-50.

Schuster, U., « Die SED-Jugendkommuniques von 1961-63. Anmerkungen zur ostdeutschen Jugendpolitik vor und nach dem Mauerbau ", Jahrbuch für zeitgeschichtliche Jugendforschung, 1994/1995, p. 58-75.

« Sex-Report: Nur wenig », Der Spiegel, 26 août 1968, n 35, p. 46-50.

Sigusch, V. et Schmidt, G., Jugendsexualität. Dokumentation einer Untersuchung, Stuttgart, Enke, 1973.

Sigusch, V., Geschichte der Sexualwissenschaft, Francfort et New York, Campus, 2008.

Stanley, L., Sex Surveyed, 1949-1994: From Mass-Observation's “Little Kinsey” to the national survey and the Hite Reports, Londres, Taylor et Francis, 1995.

Starke, K. et Friedrich, W. (dir.), Liebe und Sexualität bis 30, Berlin[-est], VEB Deutscher Verlag der Wissenschaften, 1984.

Starke, K. et Weller, K., "Partner- und Sexualforschung », in Friedrich, W., Förster, P. et Starke, K. (dir.), Das Zentralinstitut für Jugendforschung in 
Leipzig 1966-1990. Geschichte, Methoden, Erkenntnisse, Berlin, Ost, 1999, p. 396-419.

StaRKe, K., « Die ungewöhnliche gewöhnliche Sexualität in der DDR », in ELM, L., Keller, D. et MoceK, R. (dir.), Ansichten zur Geschichte der DDR, vol. 8, $2^{\mathrm{e}}$ partie, Eggersdorf, Kirchner, 1997, p. 156-200.

Starke, K., Junge Partner. Tatsachen über Liebesbeziehungen im Jugendalter, Leipzig, Jena et Berlin, Urania, 1980.

Steger, F. et Schochow, M., Disziplinierung durch Medizin. Die geschlossene Venerologische Station in der Poliklinik Mitte in Halle (Saale) 1961 bis 1982, Halle, Mitteldeutscher Verlag, 2014.

SteInbacher, S., Wie der Sex nach Deutschland kam. Der Kampf um Sittlichkeit und Anstand in der frühen Bundesrepublik, Munich, Siedler, 2011.

Süss, W., " „Rationale Politik“ durch sozialwissenschaftliche Beratung? Die Projektgruppe Regierungs- und Verwaltungsreform 1966-1975 », in FiscH, S. et Rudloff, W. (dir.), Experten und Politik. Wissenschaftliche Politikberatung in geschichtlicher Perspektive, Berlin, Duncker et Humblot, 2004, p. 329-348.

UlBricht, W., « Sozialistische Ethik und Moral », Elternhaus und Schule, 7/11, 1958, p. 15.

Vollmer, Ch., «Verliebt, geliebt und liebenswert », Bravo, 1964, n 26.

Von Friedeburg, F., Die Umfrage in der Intimsphäre, Stuttgart, Enke, 1953.

Von RöNN, P., «Die Homosexualitätsentwürfe von Hans Giese und der lange Schatten von Hans Bürger-Prinz », Z. Sexualforsch., 13, 2000, p. 277-310.

Von Soden, K., Die Sexualberatungsstellen der Weimarer Republik, 1919-1933, Berlin, Hentrich, 1988.

WeISCHER, Ch., Das Unternehmen „Empirische Sozialforschung“. Strukturen, Praktiken und Leitbilder der Sozialforschung in der Bundesrepublik Deutschland, Munich, Oldenbourg, 2004. 